\title{
Design Modeling of the 100-J Diode-Pumped Solid-State Laser for Project Mercury
}

\author{
C. Orth, R. Beach, C. Bibeau, E. Honea, \\ K. Jancaitis, J. Lawson, C. Marshall, \\ R. Sacks, K. Schaffers, J. Skidmore, S. Sutton
}

This paper was prepared for and presented at the SPIE International Symposium on High-Power Laser and Applications San Jose, California January 24-30, 1998

February 23, 1998

This is a preprint of a paper intended for publication in a journal or proceedings. Since changes may be made before publication, this preprint is made available with the understanding that it will not be cited or reproduced without the permission of the author. 


\section{DISCLAIMER}

This document was prepared as an account of work sponsored by an agency of the United States Government. Neither the United States Government nor the University of California nor any of their employees, makes any warranty, express or implied, or assumes any legal liability or responsibility for the accuracy, completeness, or usefulness of any information, apparatus, product, or process disclosed, or represents that its use would not infringe privately owned rights. Reference herein to any specific commercial product, process, or service by trade name, trademark, manufacturer, or otherwise, does not necessarily constitute or imply its endorsement, recommendation, or favoring by the United States Government or the University of California. The views and opinions of authors expressed herein do not necessarily state or reflect those of the United States Government or the University of California, and shall not be used for advertising or product endorsement purposes 


\title{
Design modeling of the 100-J diode-pumped solid-state laser for Project Mercury
}

\author{
C. Orth, ${ }^{a}$ R. Beach, C. Bibeau, E. Honea, K. Jancaitis, J. Lawson, C. Marshall, \\ R. Sacks, K. Schaffers, J. Skidmore, S. Sutton \\ Lawrence Livermore National Laboratory \\ L-490, P. O. Box 808, Livermore, CA 94550-0808
}

\begin{abstract}
We present the energy, propagation, and thermal modeling for a diode-pumped solid-state laser called Mercury being designed and built at LLNL using Yb:S-FAP [i.e., $\mathrm{Yb}^{3+}$-doped $\mathrm{Sr}_{5}\left(\mathrm{PO}_{4}\right)_{3} \mathrm{~F}$ crystals] for the gain medium. This laser is intended to produce $100 \mathrm{~J}$ pulses at 1 to $10 \mathrm{~ns}$ at $10 \mathrm{~Hz}$ with an electrical efficiency of $\sim 10 \%$. Our modeling indicates that the laser will be able to meet its performance goals.
\end{abstract}

Keywords: diode pumped, solid-state laser, Yb:S-FAP, gas cooling, deformable mirror, wavefront distortion, laser diode, lens duct, microlens.

\section{INTRODUCTION}

We have previously demonstrated diode-pumped solid-state lasers (DPSSLs) with an equivalent output of two joules or less when operating in a free-running mode. ${ }^{1,2}$ Project Mercury at the Lawrence Livermore National Laboratory (LLNL) is a project to design (FY97-98), build (FY98-99), and operate (FY00) a 100-joule 10-Hz DPSSL that is compatible with scaling to $>1$ $\mathrm{kJ}$ in future single-arm systems, and eventually to inertial fusion energy (IFE) systems at the MJ scale. The goals include an overall electrical efficiency of $10 \%$ or more, a final focus less than 5 times the size of a diffraction-limited beam, and pulse lengths of 1 to $10 \mathrm{~ns}$. The gain medium selected is Yb:S-FAP [i.e., $\mathrm{Yb}^{3+}$-doped $\mathrm{Sr}_{5}\left(\mathrm{PO}_{4}\right)_{3} \mathrm{~F}$ ] pumped at about $900 \mathrm{~nm}$, operated at $1047 \mathrm{~nm}$ for $1 \omega$, and harmonically converted to $2 \omega$ or $3 \omega$ with a conversion efficiency exceeding $50 \%$. Project Mercury is intended to provide a natural extension of the solid-state-laser technology of the National Ignition Facility (NIF) towards IFE applications. In this paper, we briefly describe the laser design, and then summarize the initial modeling in terms of the cnergy flow, the thermal management of the Yb:S-FAP crystals, and the propagation of pulses through the laser.

\section{LASER DESIGN}

The preliminary design of the laser system is shown in Figure 1. The laser system utilizes three subsystems for pulse amplification: a fiber oscillator, a regenerative amplifier (regen), and two power amplifiers. The fiber oscillator is used to generate the initial optical seed pulse with the appropriate bandwidth, pulse shape, and pulse length for optimum energy extraction.

The oscillator will be capable of producing output energies up to $10 \mathrm{~nJ}$. The output pulse will then be injected into the regen for spatial beam shaping and amplification of the pulse to 10-100 mJ. Although the design of the fiber oscillator is very similar to that used in the NIF front end, the design of the regen incorporates an advanced design with the use of a phase plate in the cavity for improving overall fill factor and energetics. The final amplification stages are accomplished through four passes of the beam through two gas-cooled amplifier head assemblies. The beam is injected off axis and near the focus to take advantage of angular multiplexing concepts which conveniently avoid the use of an optical switch (i.e. Pockels cell or Faraday rotator) at large apertures and high average power. ${ }^{3}$ After reflection from the deformable mirror at the end of pass one, the beam goes back through the same optics for pass two along a slightly different path. In this way, the beam can be re-injected by the reverser optics back into the amplifier chain for a final two passes with image relaying preserved. The deformable mirror could be placed either at the end of the amplifier path (as shown) or within the reverser optics to correct for wavefront distortions incurred during amplification.

\footnotetext{
"Further information for first author - E-mail: orth2@IInl.gov; Telephone: (925) 422-8665; FAX: (925)423-1076.
} 
Figure 2 shows a more detailed picture of the pumping geometry. The amplifier head will be optically pumped from both sides. The dual pumping design allows for more uniform pumping and thermal loading on the crystals. The diode array consists of 40 tiles with each tile containing 40 diode laser bars, each opcrating at $100 \mathrm{~W}$ peak.

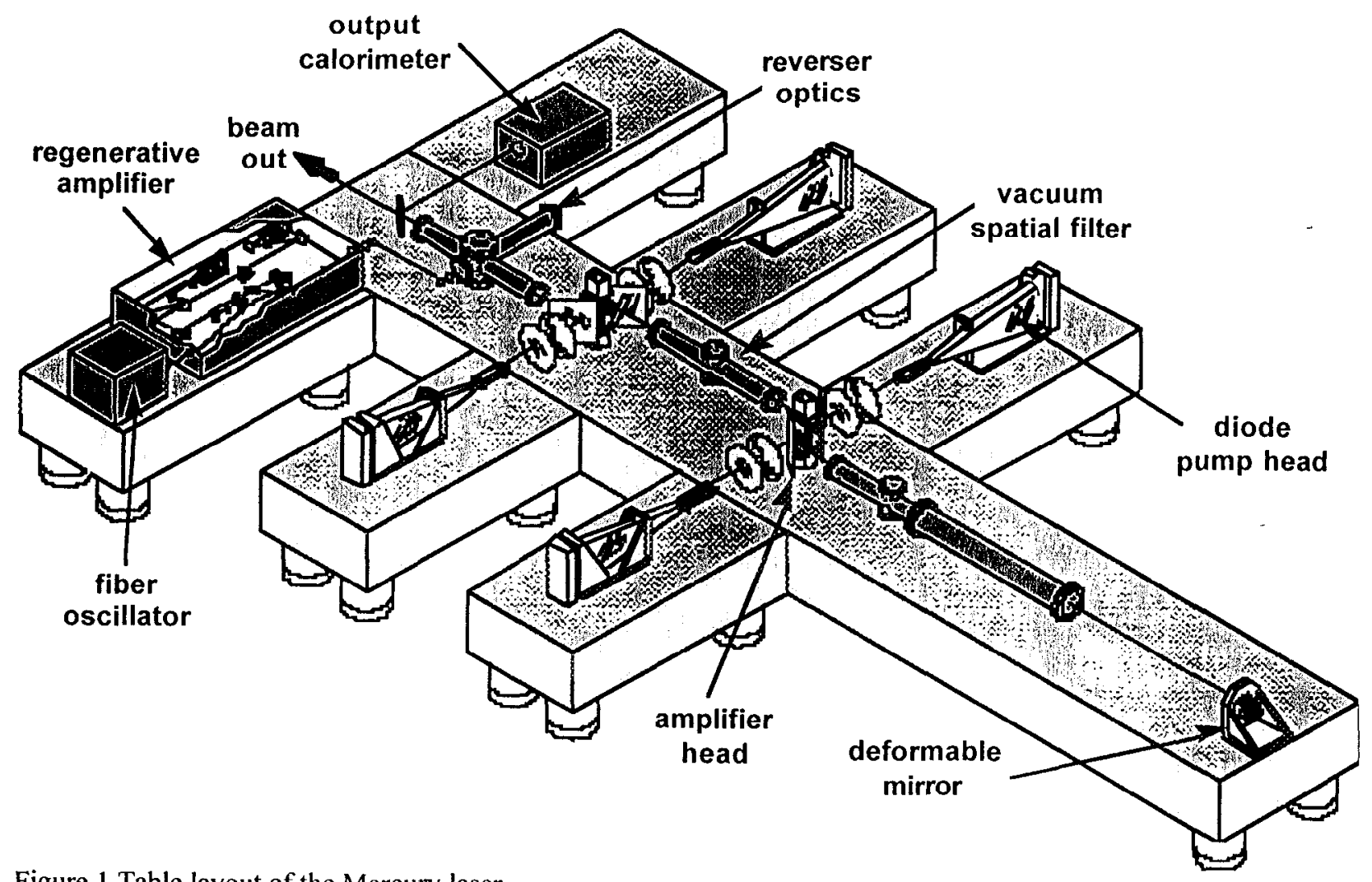

Figure 1 Table layout of the Mercury laser

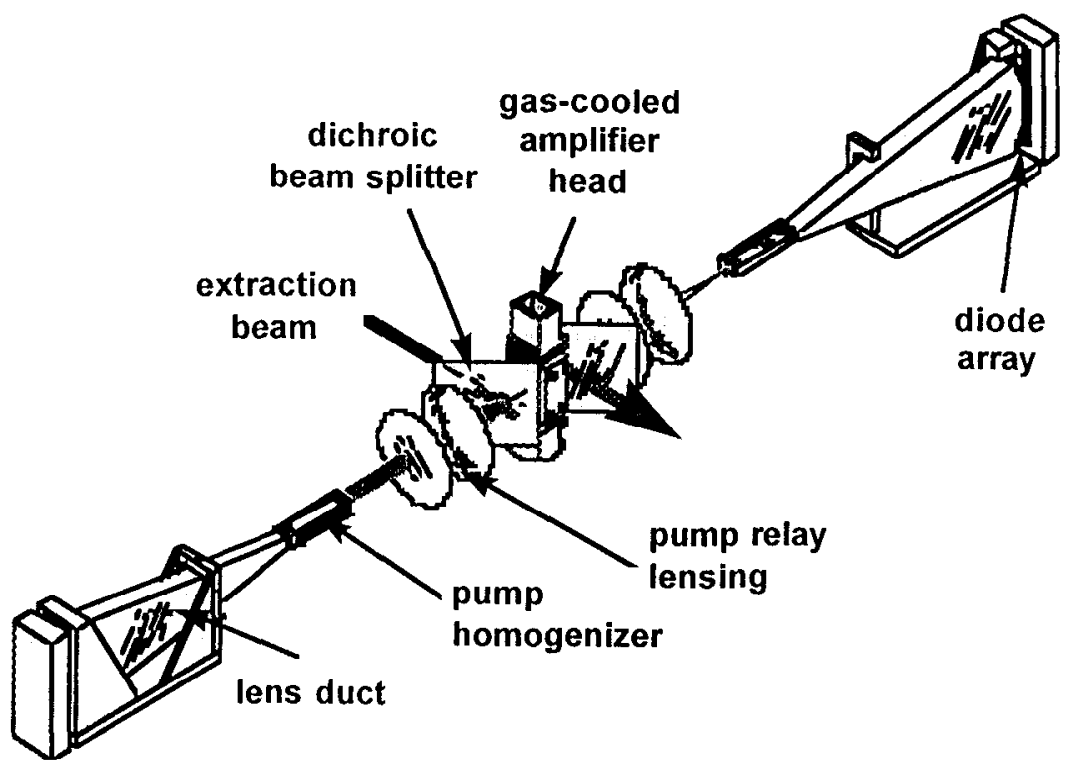

Fig. 2. Layout showing the placement of the pump delivery system and the Yb:S-FAP stack of slabs in the gas cooled head assembly. The arrow shows the path of the extraction beam. 
The light from the diode array is first condensed with a lens duct followed by a optical element which homogenizes the spatial profile of the pump beam by multiple internal reflections. The light emerging from the output of the homogenizer is relayimaged onto the gain media with a pair of lenses. The angled dichroic beam splitters allow the pump beam to pass through the optic and into the amplifier head while allowing the extraction beam to be reflected. In order to test the conceptual design shown above, we are building a prototype of the pump delivery system in FY98

\section{MODELING}

The Yb:S-FAP crystal has an absorption cross section of 5 to $9 \times 10^{-20} \mathrm{~cm}^{2}$, and is an anisotropic crystal with emission cross sections of $\sigma_{\mathrm{c}}=7.3 \times 10^{-20} \mathrm{~cm}^{2}$ and $\sigma_{\mathrm{a}}=1.5 \times 10^{-20} \mathrm{~cm}^{2}$. This medium has a bandwidth of $\sim 6 \mathrm{~nm}$ and an intrinsic storage lifetime of $1.1 \mathrm{~ms}$ (which becomes 1.3 to $1.6 \mathrm{~ms}$ with radiation trapping). Our modeling considered how 900 -nm light from laser diodes would pump this medium, how the medium would be cooled, and how laser light would propagate in four passes through the laser architecture.

\subsection{Diode Emission}

Because $\mathrm{Yb}: \mathrm{S}-\mathrm{FAP}$, like many other solid-state laser materials, has an absorption feature only a few nanometers in width, efficient operation of the system requires a correspondingly narrow diode emission, including the effects of wavelength chirp. Furthermore, power sag over the course of the pulse affects the amount of total energy delivered by the diode array. While the $1.1 \mathrm{~ms}$ storage lifetime in $\mathrm{Yb}: \mathrm{S}-\mathrm{FAP}$ is advantageous in reducing the diode power required for a given output pulse energy, the increased pulse duration exacerbates the effects of diode emission chirp and power sag. Therefore a detailed understanding of diode emission is required to maximize system performance and minimize cost.

We have developed an approximate, analytical model of diode emission which assumes one-dimensional heat flow and linear temperature dependences for chirp and electrical efficiency. ${ }^{4}$ Unlike numerical methods, ${ }^{5}$ the analytical expressions make it possible to quickly trade-off system parameters such as maximum output power, pulse duration, diode cavity length, maximum junction temperature rise and emission bandwidth tolerances. We find that the temperature rise of the diode bar is approximately described by:

$$
\Delta \mathrm{T}(\mathrm{t})=\frac{2(1-\eta) \mathrm{P}_{\mathrm{opt}}}{\sqrt{\pi} \eta \mathrm{A}} \frac{\sqrt{\mathrm{K}_{\mathrm{hs}} \kappa_{\mathrm{GaAs}}}}{\mathrm{K}_{\mathrm{hs}} \mathrm{K}_{\mathrm{GaAs}}^{1 / 2}+\mathrm{K}_{\mathrm{GaAs}} \kappa_{\mathrm{hs}}^{1 / 2}} \mathrm{t}^{1 / 2}
$$

where $P_{\text {opt }}$ is the initial optical power, $\eta$ is the electrical-optical efficiency of the laser diode bar (assumed constant during the pulse), $A=I_{\text {cavl }}$ bar is the thermal footprint, $K$ is the thermal conductivity, and $K$ is the thermal diffusivity

$$
\kappa=\frac{K}{\rho C_{p}} .
$$

of the heatsink material and GaAs diode bar substrate. For these calculations we take typical values for the cavity length, $\mathrm{l}_{\mathrm{cav}}=500 \mu \mathrm{m}$ and bar length, $\mathrm{l}_{\mathrm{bar}}=1 \mathrm{~cm}$.

Using a value of $\mathrm{d} \lambda / \mathrm{dT}=0.32 \mathrm{~nm} /{ }^{\circ} \mathrm{C}$ estimated for $900 \mathrm{~nm}$ emission, the center wavelength of the diode emission is then approximately described by,

$$
\lambda_{\mathrm{p}}(\mathrm{t})=\lambda_{\mathrm{p} 0}+(\mathrm{d} \lambda / \mathrm{dT})^{*} \Delta \mathrm{T}(\mathrm{t})
$$

Similarly, we can approximate the power sag during the pulse by

$$
\mathrm{P}(\mathrm{t})=\mathrm{P}_{0} *\left[1-(\mathrm{dP} / \mathrm{dT})^{*} \Delta \mathrm{T}(\mathrm{t})\right]
$$

where typical values of $\mathrm{dP} / \mathrm{dT}$ range from 0.5 to $1.0 \%$ /degree $\mathrm{C}$. Assuming an initial Gaussian diode emission spectrum, the emission at any time $t$ during the pulse is 


$$
P(\lambda, \Delta T)=\frac{P(\Delta T)}{a \cdot \sqrt{\pi}} e^{-\left\{\frac{\lambda-\lambda_{p}(\Delta T)}{a}\right\}^{2}}
$$

and the total encrgy delivered by the diode array as a function of wavelength is

$$
\mathrm{E}(\lambda, \tau)=\int_{0}^{\tau} \mathrm{P}(\lambda, \Delta \mathrm{T}(\mathrm{t})) \mathrm{dt}
$$

Good agreement is found between the model and experimental data from laser-diode bars mounted on $\mathrm{Si}$ and $\mathrm{BeO}$ heatsinks. With this information it is possible to perform the detailed trade-offs necessary to optimize many of the important system parameters such as pump pulse duration, the value of higher performance heatsinks and the effect on brightness of other factors such as manufacturing tolerances.

\section{$3.2 \mathrm{Yb}:$ S-FAP crystal growth \& characterization}

Significant progress has been made in understanding the growth characteristics and defect chemistry of Yb:S-FAP crystals to provide high optical quality material for Mercury. This laser requires crystalline slabs of dimension $3 \times 5 \times 0.75 \mathrm{~cm}$ for the active gain region. Growth of sufficient size crystals to provide such slabs poses a challenge because of a number of defect structures, including cloudiness in as-grown boules, core defects, slip dislocations, and cracking in boules of diameter $>4.0$ $\mathrm{cm}$. To understand each of these defects and determine a feasible growth technique for producing high optical quality crystals, a substantial effort has been initiated to study the growth of Yb:S-FAP.

After studying these defect structures in Yb:S-FAP crystals in considerable detail, we proposed and implemented methods to reduce or eliminate them. ${ }^{6}$ We eliminated cloudiness in as-grown boules by two techniques: (1) post-growth annealing over the melt for approximately three days at several hundred degrees below melting, and (2) adding $\mathrm{SrF}_{2}$ (a component making up the S-FAP structure) during the growth process to prevent the initial formation of cloudiness in the boules. This cloudiness is believed to be associated with $\mathrm{O}^{2-}$-rich defects in the crystal lattice caused by a deficiency of $\mathrm{F}^{-}$ions. Core defects can be attributed to the vaporization of $\mathrm{SrF}_{2}$ from the melt causing an unstable growth interface between the crystal and melt where impurity phases can be trapped. We have used scanning electron microscopy in conjunction with X-ray analysis (SEM-EDS) to identify the composition of the core defects as mainly $\mathrm{Yb}_{2} \mathrm{O}_{3}$, the dopant, and $\mathrm{Sr}_{3}\left(\mathrm{PO}_{4}\right)_{2}$, which are the natural decomposition by-products from the loss of $\mathrm{SrF}_{2}$ from the melt. We are now using various methods to reduce the magnitude of core defects in the crystals. The implementation of a larger diameter crucible (3" to 4") decreased the effects of $\mathrm{SrF}_{2}$ vaporization from the melt, simply by increasing the available melt volume so that small losses are relatively insignificant to the overall composition. In addition, slowing the growth rate of the boules by half has helped to stabilize the growth interface allowing for a reduction of trapped impurity phases. Cracking has become a very important issue for larger diameter crystals $(>4.0 \mathrm{~cm})$ that are more sensitive to thermal gradients and stresses. We solved this problem by careful consideration of the crystal growth furnace design to produce low thermal gradients for the growth of crack-free crystals; low gradients are also advantageous for controlling the core defect problem.

Another critical issue associated with Yb:S-FAP crystals is that of slip dislocations. This phenomena appears as slight changes in the refractive index of the crystal or waves running through the crystal with a "columnar-type" orientation perpendicular to the $c$-axis. These dislocations are formed by propagation from the seed to the crystal and strain-induced deformations caused by an unstable growth interface. Reducing the number of slip dislocations requires using "dislocation-free" secds and careful consideration of the growth rate and interface shape to stabilize growth-preventing strain in the crystals.

At this time, significant progress has been made in understanding and controlling each of the defect structures in an effort to develop the growth of large diameter, high optical quality Yb:S-FAP crystals. Current growth capabilities have produced the first $>5.0 \mathrm{~cm}$ diameter crystals with limited defects (Fig. 3).

Figure 3. Picture of a 5.0-cm low-defect Yb:S-FAP crystal.

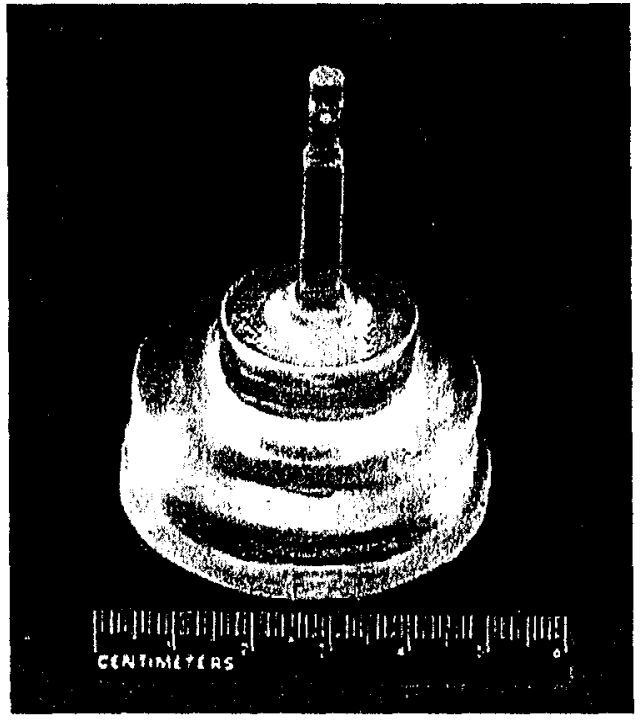




\subsection{Baseline Performance}

We have developed a baseline performance code to model energy flow in the Mercury laser system. This modeling code was developed to perform quick optimization and tradeoff studies to help zero in on a system design prior to the completion of a full physics modeling capability for the system. To keep the analysis manageable, several approximations and simplifications have been made in describing the pump excitation and laser energy extraction physics as detailed below.

To begin, we model the laser diode pump array performance including the effects of transient heating during the laser diode pump pulsc. Transicnt diode heating is accounted for by dynamically tracking the roll-off in diode array output power, and the red shift in the diode emission spectrum, with increasing temperature during the pulse. In addition to the contribution of diode chirp to the pump spectrum, the entire array is characterized by a Gaussian distribution of wavelengths representing the spread in the center wavelengths of the approximately 6400 individual diode bars that will comprise the entire pump array. The peak power capability of the array is $640 \mathrm{~kW}$. The following equation is used to describe the temporal and spectral dependence of the diode array output energy

$$
\frac{\partial^{2} E}{\partial t \partial \lambda}=\frac{P(t)}{\sqrt{\pi} \lambda_{1 / \text { eHW }}} e^{-\left(\frac{\lambda-\lambda_{p}(t)}{\lambda_{1 / e H W}}\right)^{2}}
$$

where $\lambda_{1 / \mathrm{eHW}}$ is the $1 / \mathrm{e}$ spectral halfwidth that describes the pump array radiation and $\lambda_{\mathrm{p}}(\mathrm{t})$ is the instantaneous center wavelength of the pump pulse at time t. A thermal diffusion model that is anchored to experimental data is used to describe the transient temperature rise experienced by the diode array during a pump pulse. Calling $\Delta T(t)$ the transient temperature rise of the diodes during a pump pulse, the instantaneous central wavelength of the diode array, $\lambda_{p}(t)$, and the instantaneous optical power developed by the array, $P(t)$, are given by Eqs. (3) and (4), respectively, where $d \lambda / d T$ and $\beta=d P / d T$ are constants that characterize the diode bars used in the pump array. The transient temperature rise is given by Eq. (1), which can be put in the form,

$$
\Delta T(t)=\alpha \sqrt{t}
$$

where $\alpha$ is a constant characteristic of the diffusion properties of the BeO backplane to which the diode bars are bonded. Table 1 lists the values of the various constants that describe the diode pump array in the modeling that follows.

Table 1 Parameter values used in diode pump pulse model

\begin{tabular}{|c|c|}
\hline$\lambda_{1 / \mathrm{eHW}}$ & $4.8 \mathrm{~nm}$ \\
\hline $\mathrm{P}_{0}$ & $640 \mathrm{~kW}$ \\
\hline $\mathrm{d} \lambda / \mathrm{dT}$ & $0.3 \mathrm{~nm} /{ }^{\circ} \mathrm{C}$ \\
\hline$\alpha$ & $632^{\circ} \mathrm{C} / \mathrm{sec}^{1 / 2}$ \\
\hline$\beta$ & $0.00385 /{ }^{\circ} \mathrm{C}$ \\
\hline
\end{tabular}

Our modeling code next treats the transport of the laser diode array pump radiation into the Yb:S-FAP slabs by tracking pump radiation rays through a lens duct and homogenizer. One of the critical parameters that characterizes the laser diode array performance for the pump delivery architecture that is being pursued here is the divergence of the radiation in both the slow and fast axis directions. To control the fast axis divergence, each diode bar has its output radiation conditioned by a shaped cylindrical microlens. ${ }^{7,8}$

Figure 4 depicts measured slow and fast axis intensity profiles taken from a 40 bar prototype module that will be used in the pump diode array. The microlens conditioned fast axis divergence is characterized by a FWHM of 10 mRad in the slow axis and $150 \mathrm{mRad}$ in the fast axis. After the pump light is conditioned by the shaped cylindrical microlenses, it is delivered to a rectangular aperture at the end of a lens duct ${ }^{9}$ having the same dimensions as the slab aperture which is to be pumped. Because efficiency is the driving consideration in the design of the laser system, the lens duct is designed to have maximum transfer efficiency without regard for the uniformity of the radiation at its end. However, it is critical that the slabs be uniformly pumped to enable the extraction of a high-quality laser beam, which requires a uniform intensity pump beam. To accomplish this, the pump radiation at the lens duct output end is homogenized by being transported a short distance through a rectangular cross section waveguide having the same transverse dimensions as the lens duct output face and the slab aperture that is to be excited. The waveguide is designed such that the transmitted pump light makes several reflections with its sides in the process 


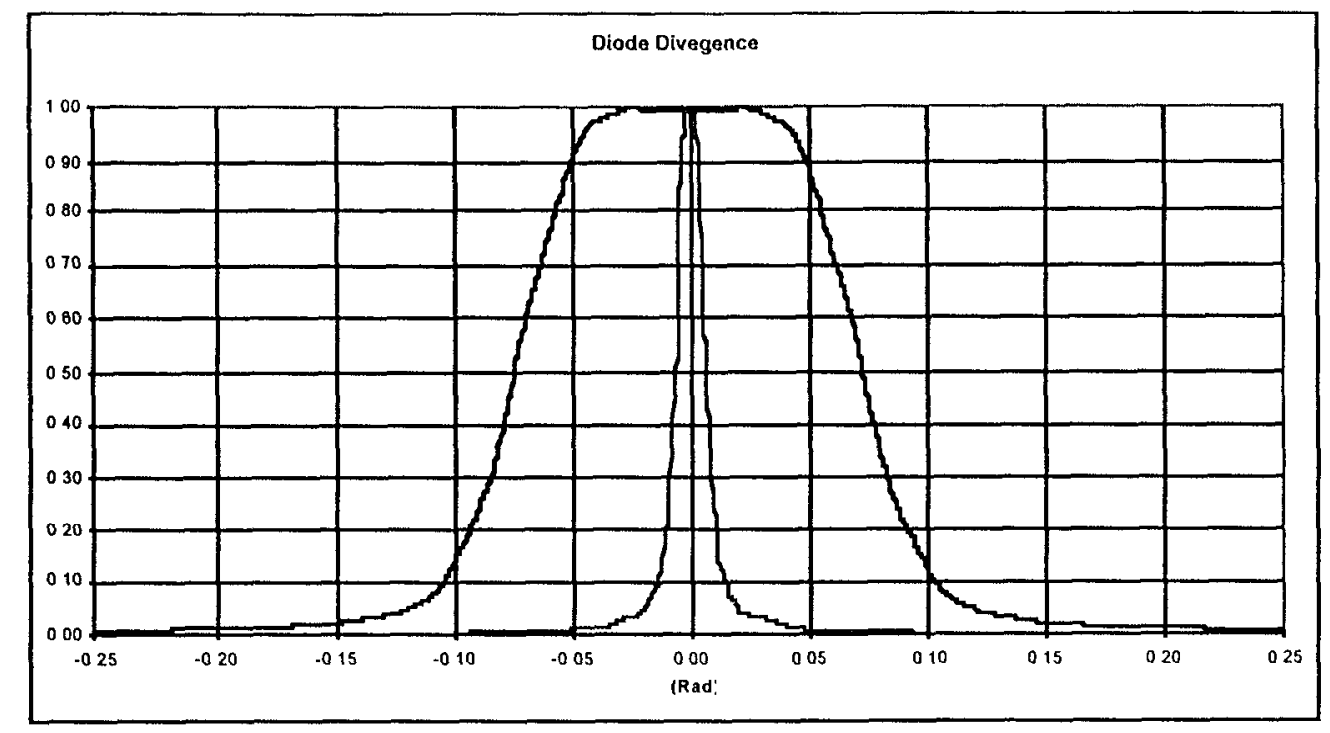

Fig 4. Slow and fast axis farfield intensity profiles from a 40 diode bar stack of laser diode arrays.
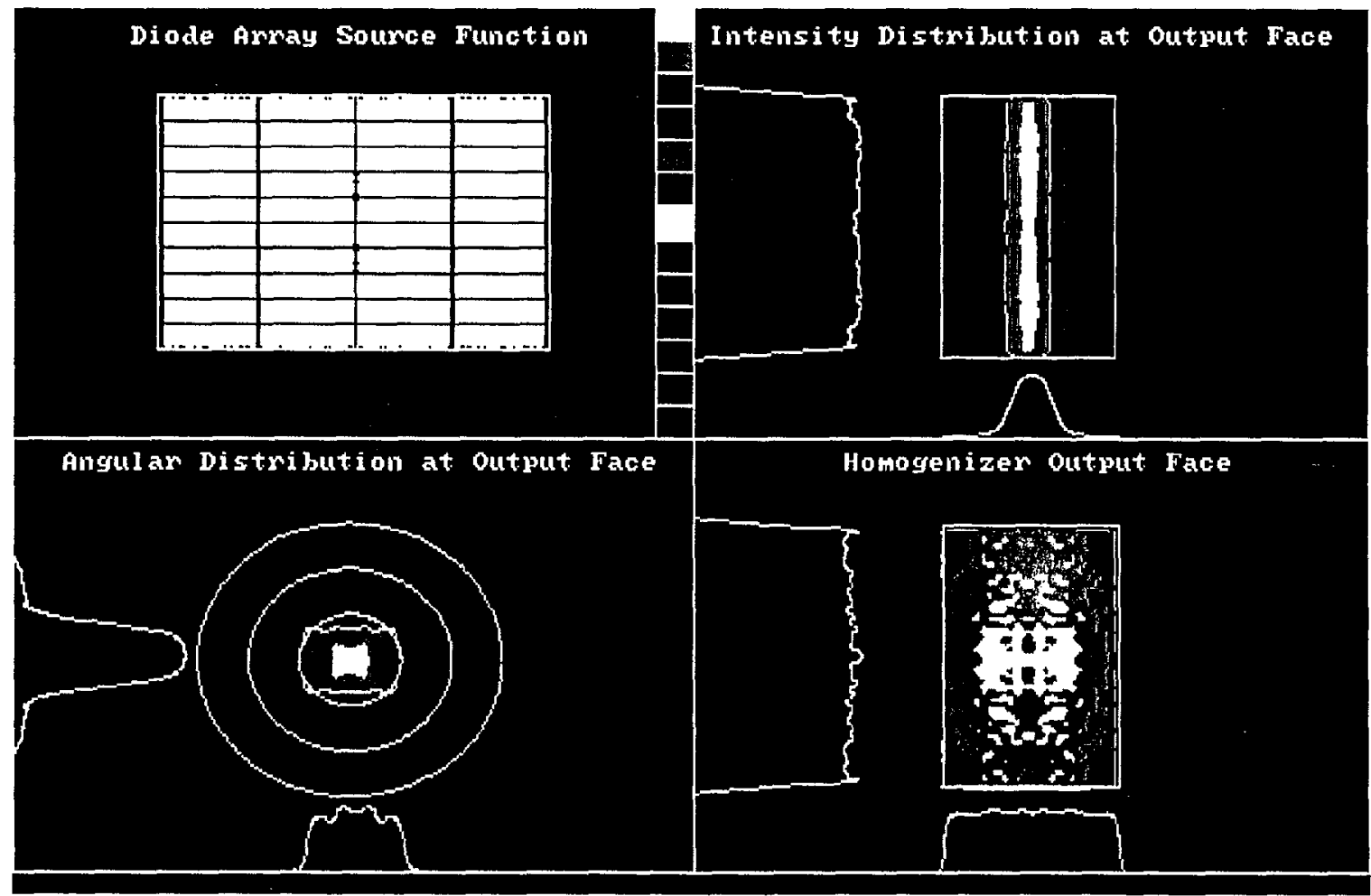

Fig. 5. Pump intensity at various locations in the pump chain. Clockwise from upper left: (1) The diode pump array. (2) The intensity at the output of the lens duct. (3) The angular distribution of the light that exits the lens duct. The inner circle is $15^{\circ}$ from the optic axis, the next circle is at $30^{\circ}$, and the output circle at $45^{\circ}$.

of rattling down its length. These reflections effectively homogenize the pump light intensity across the waveguide aperture by the time the light reaches its output end. The pump light emerging from the output end of the homogenizer is then imaged 
onto the first slab in the stack of seven slabs that are housed in the gas-cooled amplifier head. Figure 5 shows the irradiance at various places in the pump chain.

The code then forward propagates the pump radiation through the 7 slabs comprising the gain module to calculate 2dimensional pump deposition profiles at each slab, as well as slab edge-cladding optical loading values. This last portion of the code does not yet fully treat all of the physics associated with the pump excitation process as only linear absorption is assumed for the ray trace portion of the code, and effects such as amplified spontaneous emission are not treated in a spatially dependent manner. To address these shortcomings of the ray-trace portion of the modeling code, a 1-dimensional pump pulsc propagation model has been developed. This propagation code dynamically calculates the gain loading of the individual laser slabs using a treatment that includes the effects of $\mathrm{Yb}^{3+}$ ground state depletion, spectrally selective pump wave depletion, $\mathrm{Yb}^{3+}$ fluorescence decay, and amplified spontaneous emission which is handled using a simplified treatment. The following equations are used to model the double-ended pump excitation on the slabs. ${ }^{10}$

$$
\begin{aligned}
& \frac{\partial I_{r}(z, t)}{\partial z}+\frac{n}{c} \frac{\partial I_{r}(z, t)}{\partial t}=I_{1} \sigma_{p}\left[\left(f_{a}^{p}+f_{b}^{p}\right) n_{2}-f_{a}^{p} n_{0}\right] \\
& -\frac{\partial I_{l}(z, t)}{\partial z}+\frac{n}{c} \frac{\partial I_{l}(z, t)}{\partial t}=I_{l} \sigma_{p}\left[\left(f_{a}^{p}+f_{b}^{p}\right) n_{2}-f_{a}^{p} n_{0}\right] \\
& \frac{\partial n_{2}(z, t)}{\partial t}=-\frac{1}{h v_{p}}\left[I_{r}(z, t)+I_{l}(z, t)\right] \sigma_{p}\left[\left(f_{a}^{p}+f_{b}^{p}\right) n_{2}-f_{a}^{p} n_{0}\right]-\frac{n_{2}(z, t)}{\tau(t)}
\end{aligned}
$$

Where $I_{l}$ and $I_{r}$ represent the left and right propagating pump waves, respectively, $n$ is the refractive index of S-FAP, $n_{2}$ is the $\mathrm{Yb}^{3+2} \mathrm{~F}_{5 / 2}$ excited manifold number density, $\sigma_{\mathrm{p}}$ is the spectroscopic pump absorption cross section, and $\mathrm{f}_{\mathrm{a}}^{\mathrm{p}}=0.81$ and $\mathrm{f}_{\mathrm{b}}{ }^{\mathrm{p}}$ $=0.0098$ are the ${ }^{2} F_{7 / 2}$ and ${ }^{2} F_{5 / 2}$ Boltzmann occupation factors of the initial and final Stark levels coupled by the pump radiation, respectively. The output of this 1-dimensional code is used to normalize the 2-dimensional energy deposition profiles calculated using our ray trace code. As an example of the code's output, Fig. 6 depicts the gain loading in one of the slabs. The assumed cross sectional dimensions of the slabs are $4 \mathrm{~cm}$ by $6 \mathrm{~cm}$ and they are $0.75 \mathrm{~cm}$ thick. Only the central 3 $\mathrm{cm}$ by $5 \mathrm{~cm}$ is excited by the pump beam leaving a $5 \mathrm{~mm}$ skirt around the perimeter of the slab to allow the slab edge cladding to be spatially separated from the high intensity pump radiation. Another feature that we account for in our modeling is the geometrical overlap of the left and right counter-propagating pump beams with the extracted area of the slabs in each stack. Figure 7 shows schematically the pump geometry that we are using. The left going pump is imaged onto the extracted region in the right most slab and the right going pump is imaged onto the extracted region in the left most slab. This results

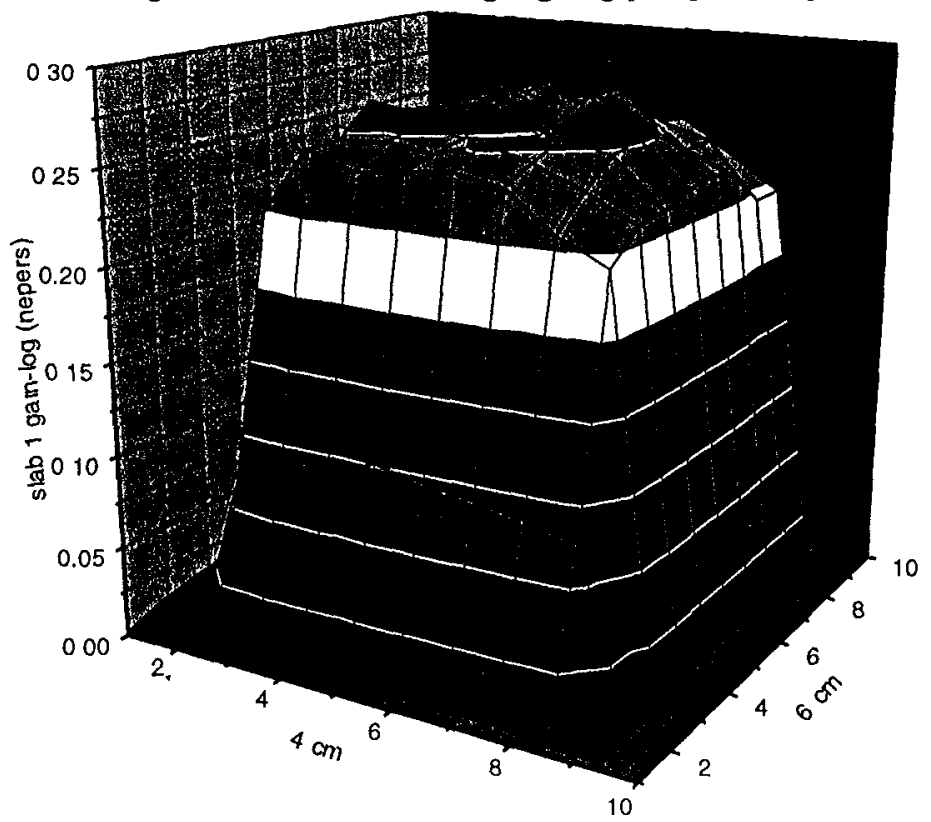
in the pump beams diffracting out of the extracted regions as they propagate away from the outermost slab on which they were imaged. The 3-D ray trace code allows us to quantify the fractional pump deposition within the slab-extracted area on a slab by slab basis. Figure 8 plots the values of these overlap integrals for the 7 slabs comprising one of the stacks.

Fig. 6. 2-D pump deposition profiles of the $\mathrm{Yb}: \mathrm{S}-\mathrm{FAP}$ are calculated using our 3-D ray trace code and 1-D pump excitation code. 


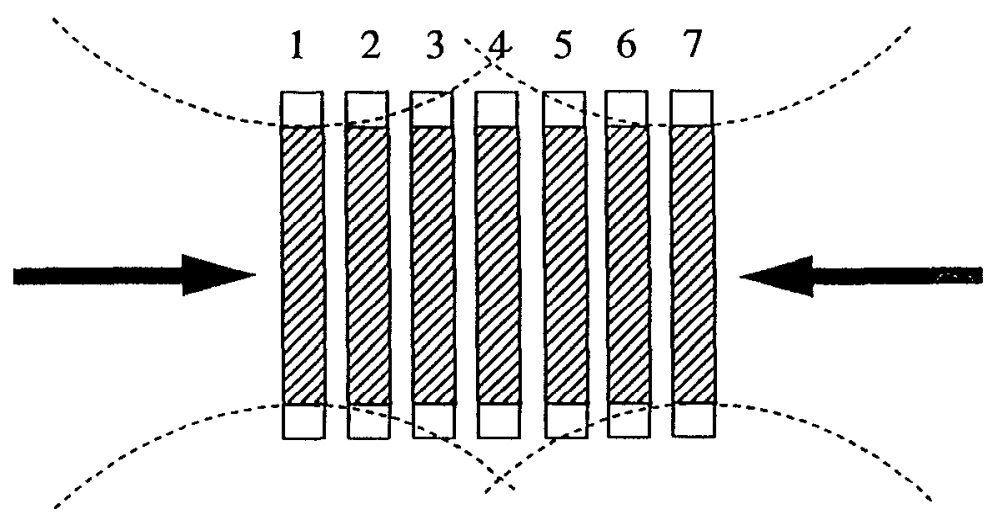

Fig. 7. The pump beams are imaged onto the extracted area of the outermost slabs but then diverge as they propagate through the remaining slabs. This divergence is shown here exaggerated for illustrative purposes; the actual divergence is less than shown.

\section{Geometric overlap of pump light with extraction area}

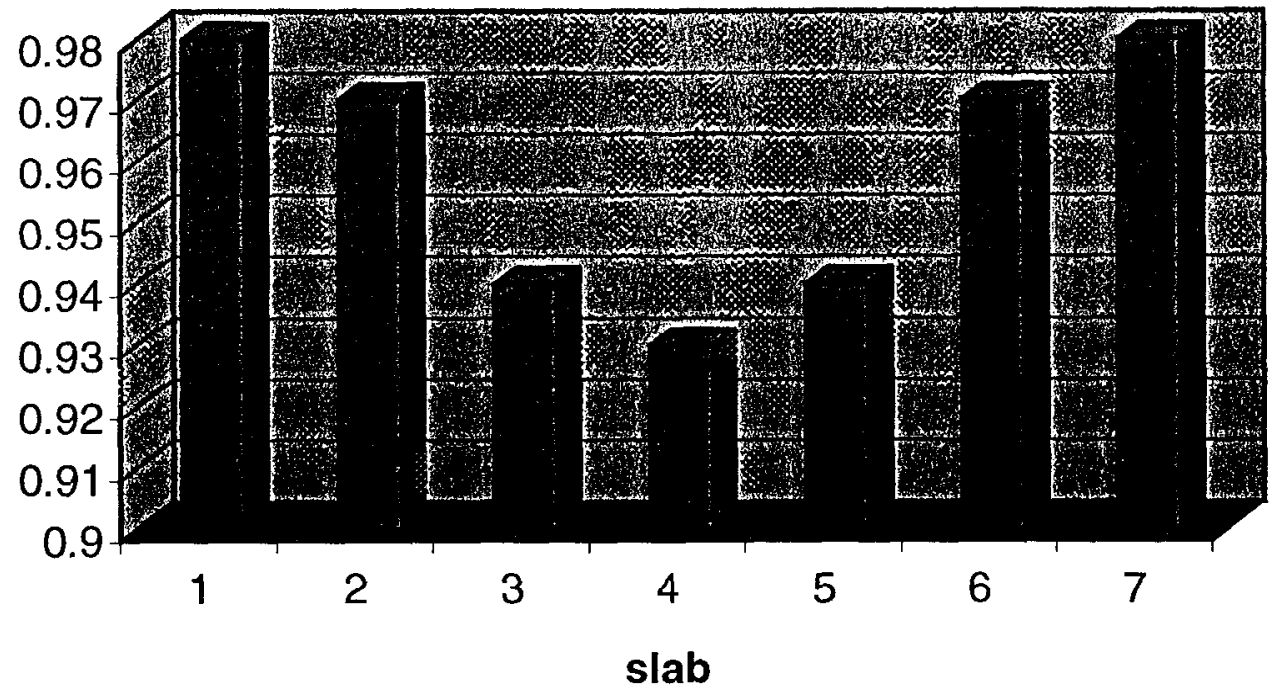

Fig. 8. Fractional pump deposition within the defined area of each slab, or the overlap integral, as calculated by our 3-D pump propagation code.

As already mentioned, our pump propagation code contains a 1-D analysis to more fully describe the physics than is done with our 3-D ray trace code. For example, the 1-D code includes spectral profiles of the pump that are dynamically updated as it is propagated through the stack of slabs, as well as the spectral profiles of the $\mathrm{Yb}^{3+}: \mathrm{S}-\mathrm{FAP}$ absorption. The spectral profile of the generated pump radiation is calculated using the above equations. This ability to model the pump radiation and its absorption 
in the slabs in some detail has turned out to be an important lever in the optimization of the laser system, primarily due to the relatively narrow pump absorption line that is being utilized in the Yb:S-FAP excitation as shown in Fig. 9. This narrow absorption feature critically determines the center wavelength of the laser diode array required for system optimization. As an example, Fig. 10 depicts the instantaneous pump power spectra generated by the laser diode array at both the beginning and end of a $1 \mathrm{~ms}$ long pump pulse for an array with optimized wavelength. In accordance with the previously discussed model there is both a red shift and a roll-off in output power evident between the beginning and the end of the pulse. Figure 11 shows one impact of the narrow absorption feature on the pump excitation radiation. The integrated pump power spectrum after the slabs exhibits a spectral hole burned into it by the absorption of the $\mathrm{Yb}^{3+}:$ S-FAP. Because one of the driving design considerations of the laser system is the optimization of its efficiency, the developed code has been very useful in specifying desired diode operating characteristics as well as performing system sensitivity studies of these diode characteristics.

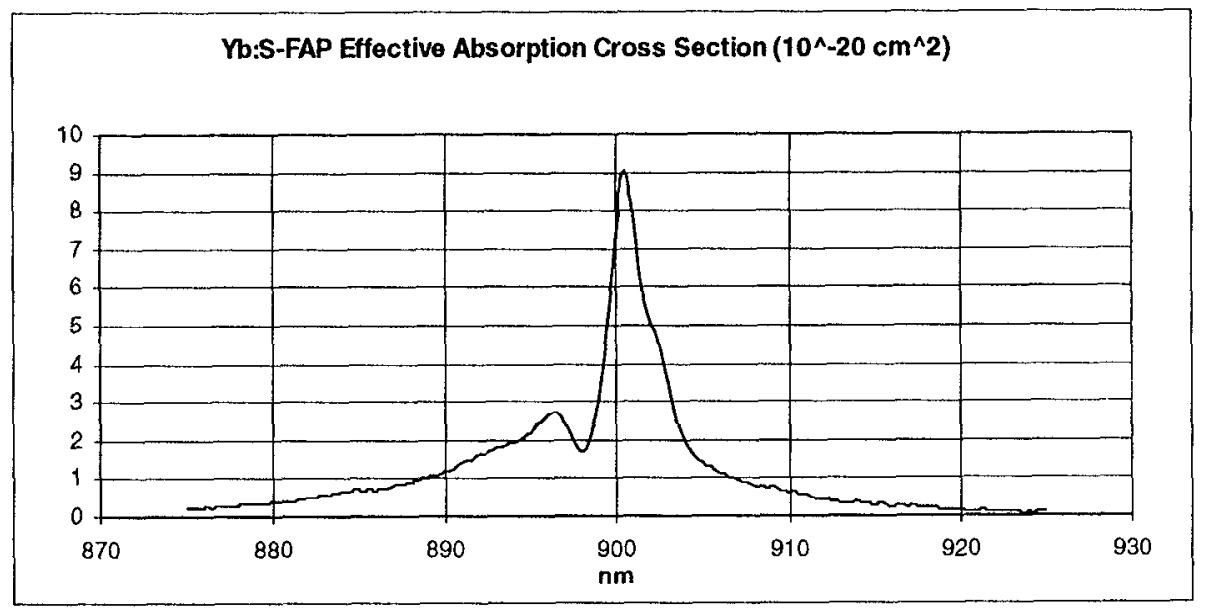

Fig. 9. $\mathrm{Yb}^{3+}:$ S-FAP effective absorption cross section.

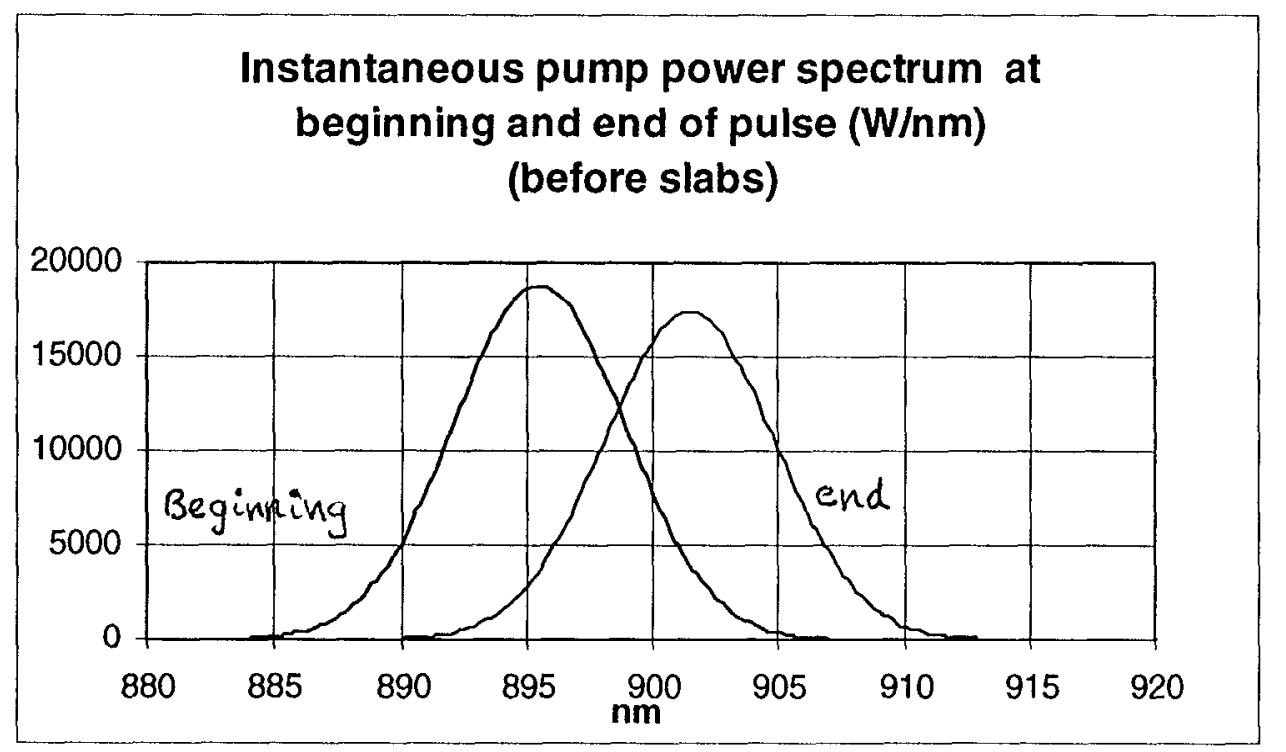

Fig. 10. Instantaneous pump power spectrum at the beginning and end of a $1 \mathrm{~ms}$ long pump pulse. 


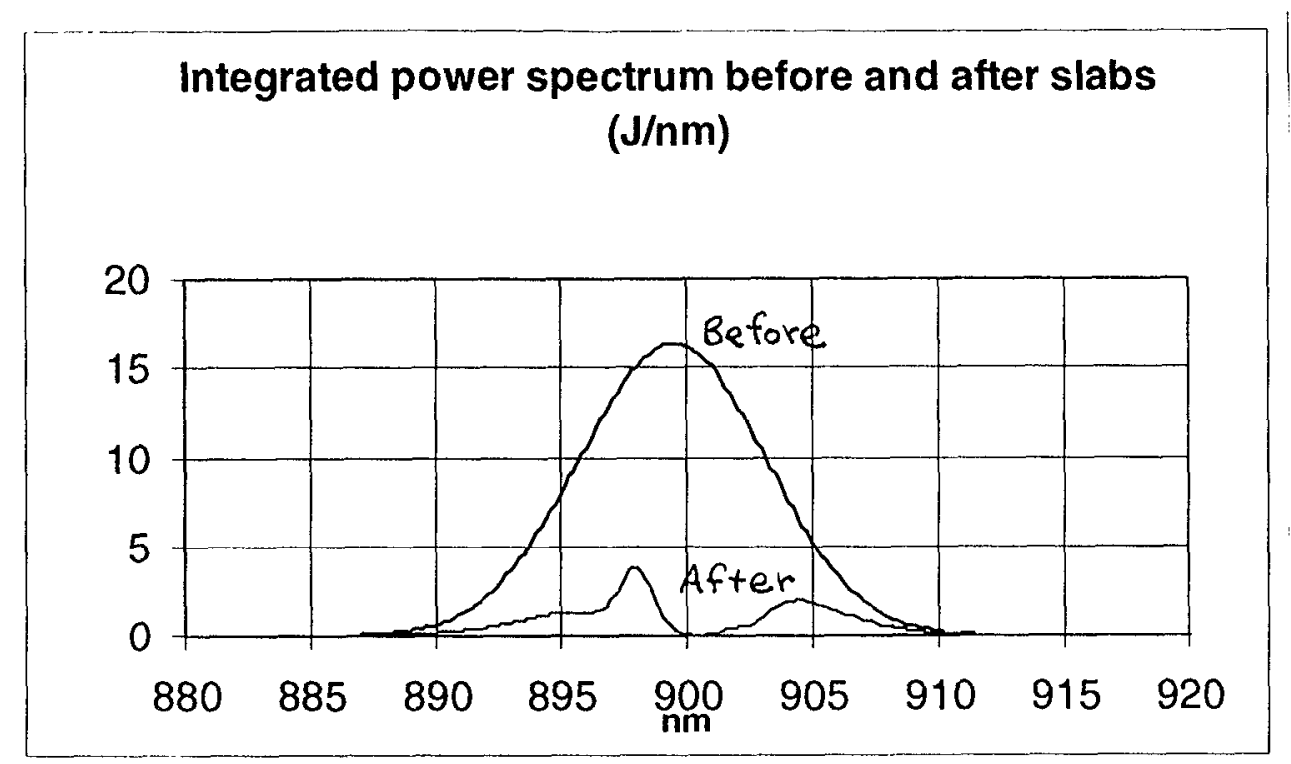

Fig. 11. Integrated pump power spectrum before and after slabs.

One of the issues that we have addressed with our modeling capability is the specification of the slab to slab dopings to both optimize laser performance and keep the thermal load from slab to slab balanced. The balancing of the thermal load from slab to slab is an important consideration for the optimum performance of the gas cooling being employed here. By using three different doping values of $1.4 \times 10^{19} / \mathrm{cm}^{3}, 1.7 \times 10^{19} / \mathrm{cm}^{3}$, and $2 \times 10^{19} / \mathrm{cm}^{3}$ as shown in Fig. 12 it is possible to hold the thermal loading from slab to slab to better than $10 \%$ as shown in Fig. 13.

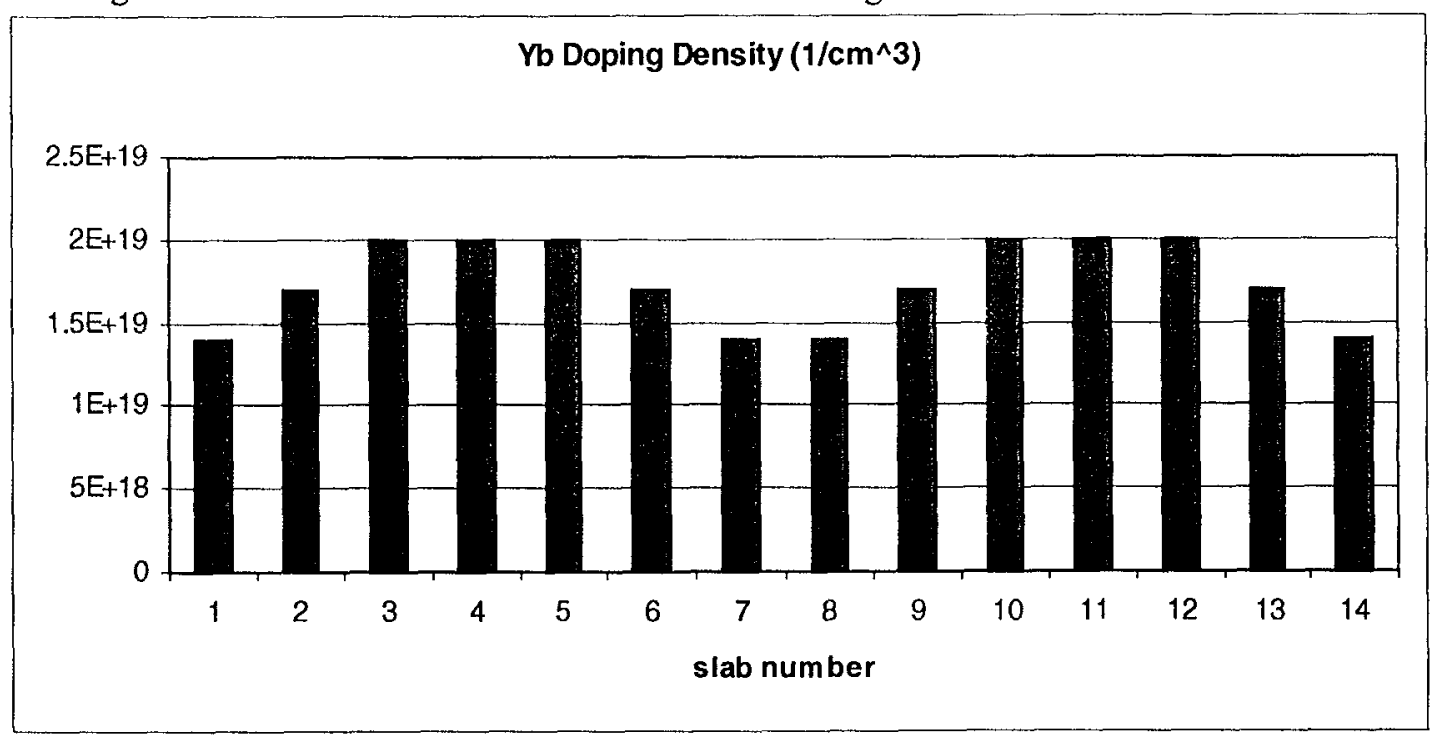

Fig. 12. $\mathrm{Yb}^{3+}$ doping densities for the fourtecn slabs that make up the Mercury laser amplifier.

Energy extraction modeling uses a standard Frantz-Nodvik analysis that has been modified to account for the quasi-three level nature of the $\mathrm{Yb}^{3+}$ ion to calculate the gain-log $\mathrm{g}_{i}$, and fluence $\Gamma_{\mathrm{i}}$ after each pass through the system.

$$
\begin{aligned}
& g_{i}=-\ln \left[1-e^{-\Gamma_{i-1} / \Gamma_{s a t}}\left(1-e^{-g_{i-1}}\right)\right] \\
& \Gamma_{i}=\Gamma_{s a t} \ln \left[1+e^{+g_{i}}\left(e^{+\Gamma_{i-1} / \Gamma_{s a t}}-1\right)\right]
\end{aligned}
$$




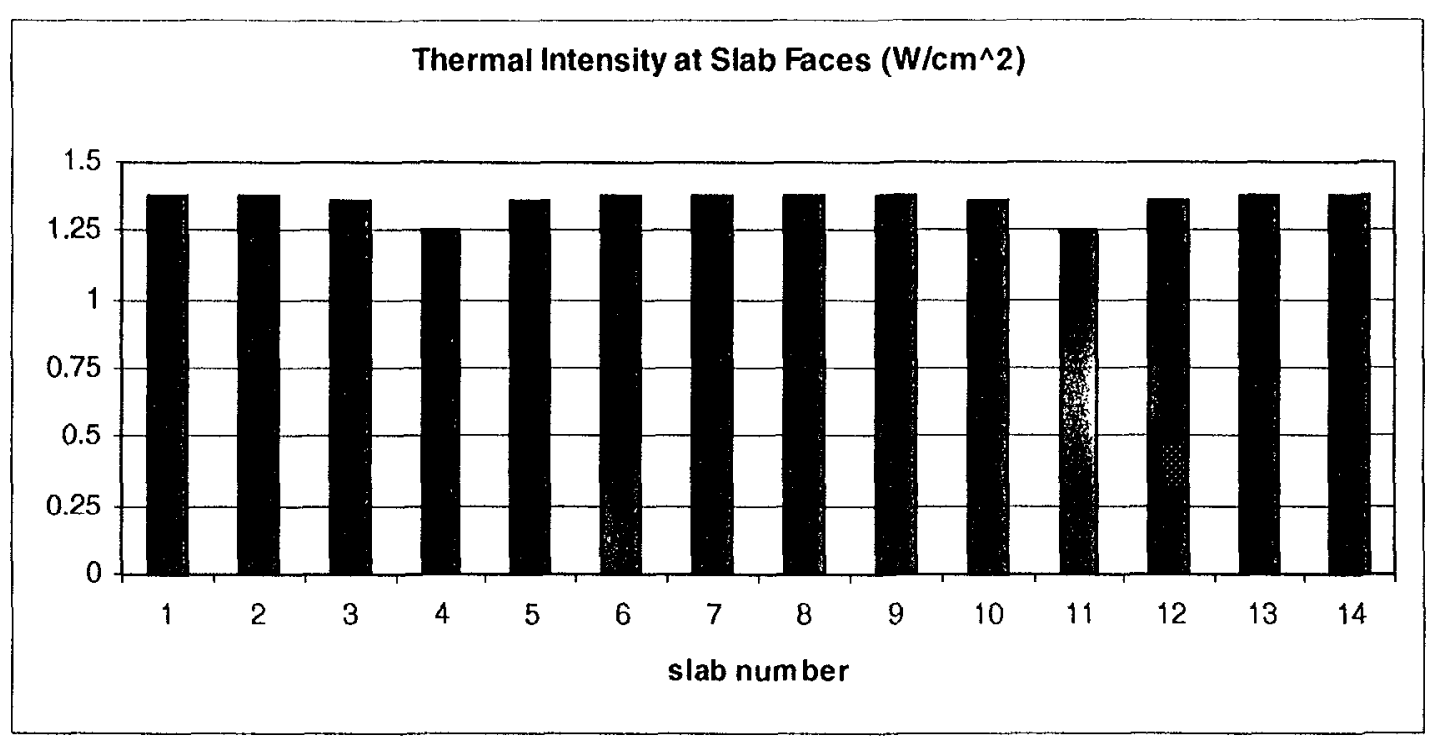

Fig. 13. Thermal intensity at slab faces with the laser operating at full average power (10 Hz PRF).

where

$$
\begin{aligned}
& g=\left[\left(f_{a}^{e}+f_{b}^{e}\right) n_{2}-f_{a}^{e} n_{0}\right] \sigma_{e m} \ell \\
& \Gamma_{s a t}=\frac{1}{f_{a}^{e}+f_{b}^{e}} \frac{h v_{e}}{\sigma_{e m t}}
\end{aligned}
$$

In (11), $\sigma_{e m}$ is the spectroscopic emission cross section of the $1047 \mathrm{~nm} \mathrm{Yb}: S-F A P$ line, which has a value of $6 \times 10^{-20} \mathrm{~cm}^{2}$ and $\mathrm{f}_{\mathrm{a}}{ }^{\mathrm{l}}$ and $\mathrm{fb}_{\mathrm{b}}{ }^{\mathrm{l}}$ are the ${ }^{2} \mathrm{~F}_{7 / 2}$ and ${ }^{2} \mathrm{~F}_{5 / 2}$ Boltzmann occupation factors of the Stark levels coupled by the laser radiation, respectively. Figure 14 shows schematically the path followed by the extracting laser pulse through the two stacks of seven slabs as it four-passes the system.

Fig. 14. Schematic diagram of the path followed by the laser extracting pulse as it passes four times through the two stacks of seven slabs each that comprise the amplifier system.

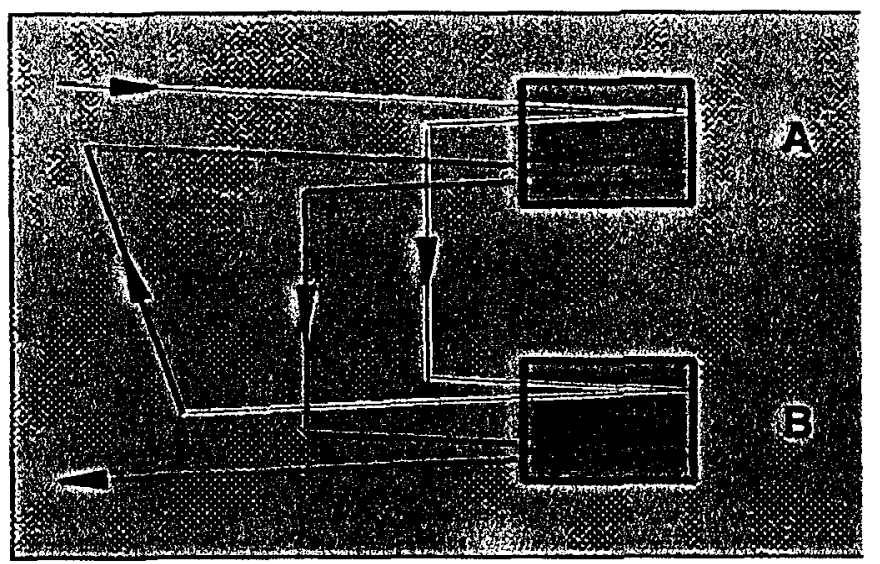



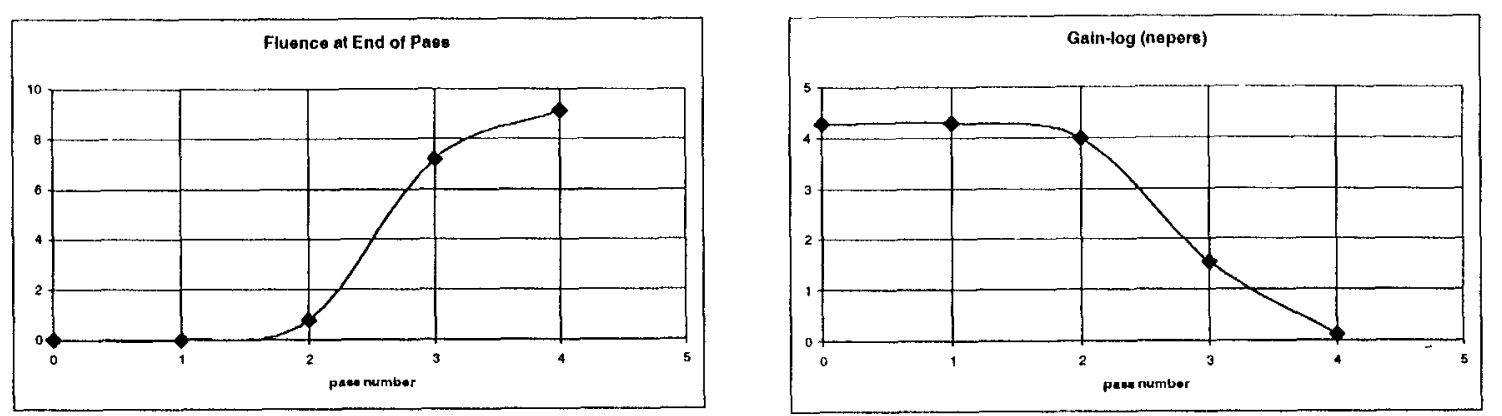

Fig. 15. Extracting fluence and net gain-log of the 14 slabs that comprise the amplifier system as a function of the pass number during the four-pass extraction.

Figure 15 shows the extracting pulse fluence and the net gain-log of the 14 slabs as a function of the pass number during the four-pass extraction. The final slab to be traversed by the extracting pulse is projected to see a fluence of $9 \mathrm{~J} / \mathrm{cm}^{2}$ underscoring the importance of high damage threshold optical coatings. In addition to tracking the extracting fluence and the gain-log as a function of pass number, the code also performs a path integral of the local extraction pulse irradiance, assuming temporally square extracting pulses, which is in turn used to calculate a B-integral for the system.

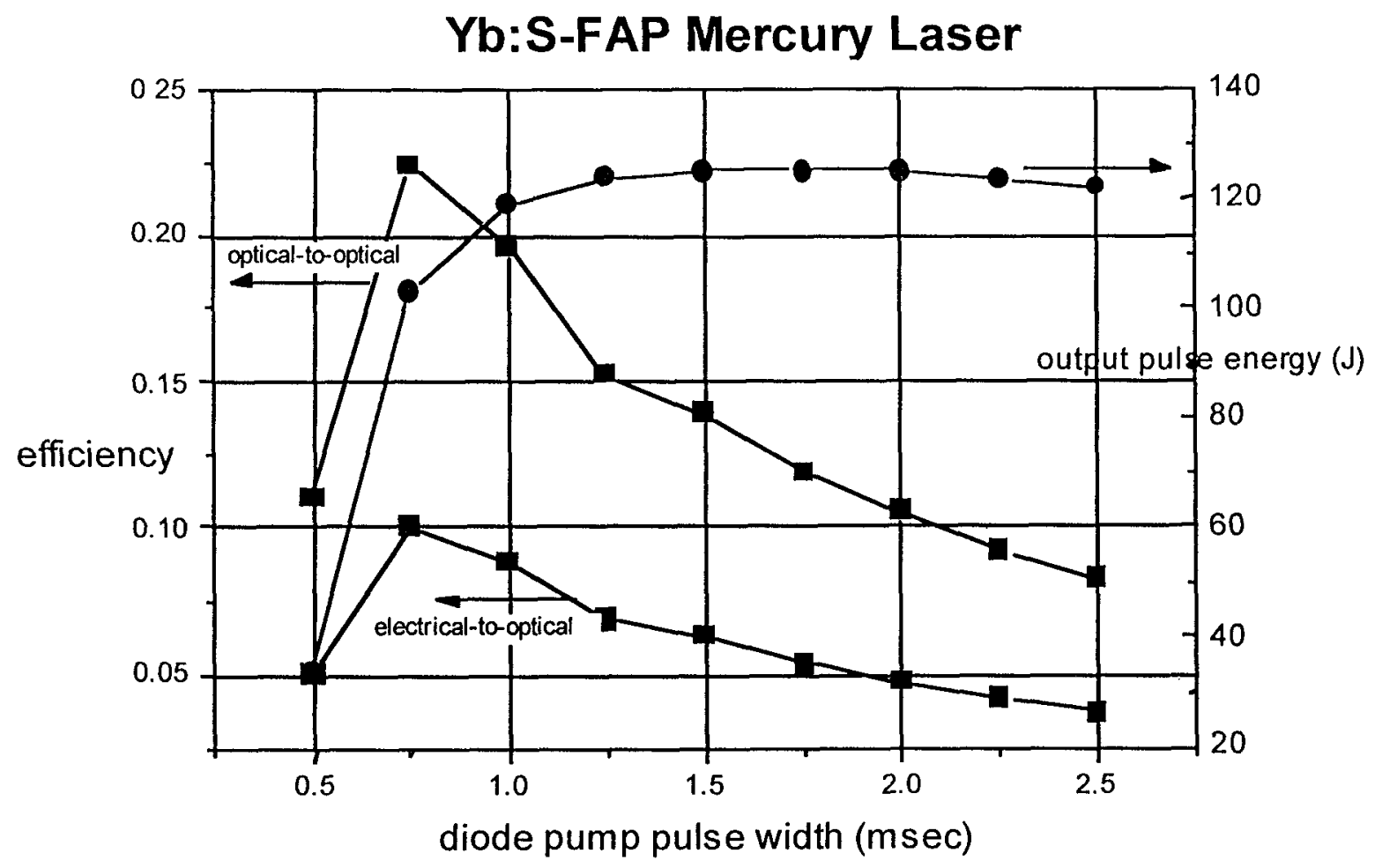

Fig. 16. Mercury laser system performance versus diode pump pulse duration. An assumed diode wall plug efficiency of $45 \%$ (electrical-to-optical) was used in generating the electrical-to- $1047 \mathrm{~nm}$ optical efficiency curve. 
As already mentioned one of the motivations for developing this preliminary energetics model for the Mercury laser system is to have the ability to perform quick sensitivity and optimizations studies. One example of a sensitivity study is shown in Fig. 16 where both efficiency and output pulse energy are plotted against the pump pulse duration. In terms of pulse energy output and overall efficiency, the goal of the Mercury laser system is $100 \mathrm{~J}$ and a wallplug efficiency of $10 \%$. Using an assumed diode efficiency (electrical-to-optical) of $45 \%$, these goals can be met with a pump pulse duration of $750 \mu \mathrm{s}$.

Another function that can be performed with the initial energetics code that we have developed is to scope out the energy flow down in the system, i.c., the various channels in which the generated pump light flows out of the Mercury laser system. Using the pie chart in Fig. 17 to represent the total optical pump energy developed by the laser diode pump arrays in a $1 \mathrm{~ms}$ pump pulse, the breakout of major energy channels is as shown.

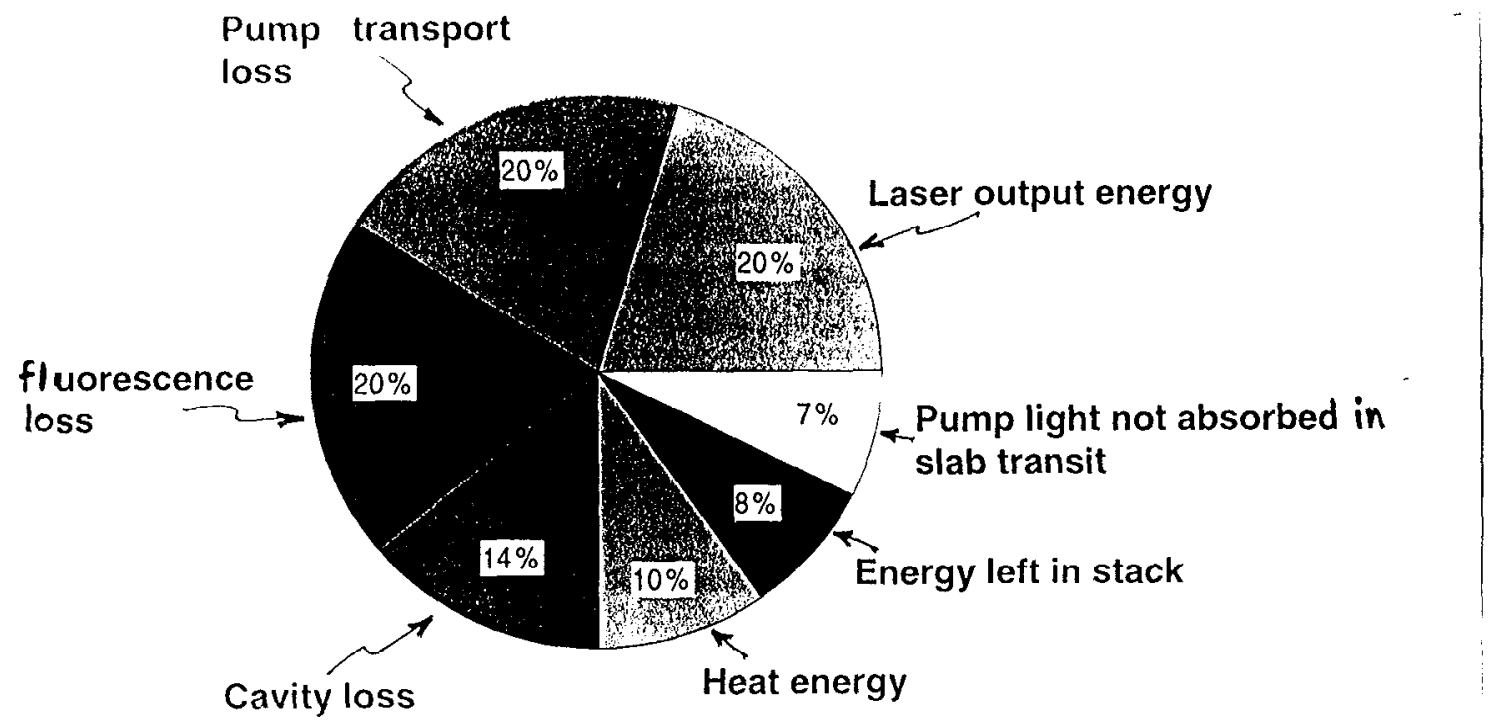

Fig. 17. The energy channels for the pump energy developed in a $1 \mathrm{~ms}$ long pump pulse.

\subsection{Optical wavefront distortions modeling}

We measured an actual crystal of Yb:S-FAP on a phase-stepping interferometer using a P-HOM technique." The P-HOM technique uses measurements of the reflected wavefront from both surfaces to determine the bulk phase properties of a sample from the transmitted wavefront. We computed the power spectral density ${ }^{12}$ (PSD) of the data (Fig. 18) excluding (i.e., in a patch outside of the core defect. The overall shape of the ID PSD is similar to that seen in many optics, although it does not follow a $v^{-155}$ power law scaling as closely as most. However, the magnitude of the ID PSD and of the wavefront rms is within a factor of 2 of the power law desired for Mercury (shown as a solid line).

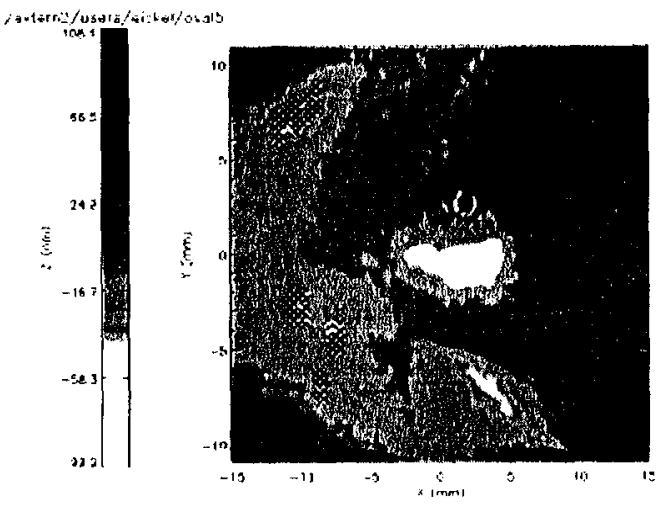

Figure 18 (a)Phase map of Yb:S-FAP crystal showing a central core defect.

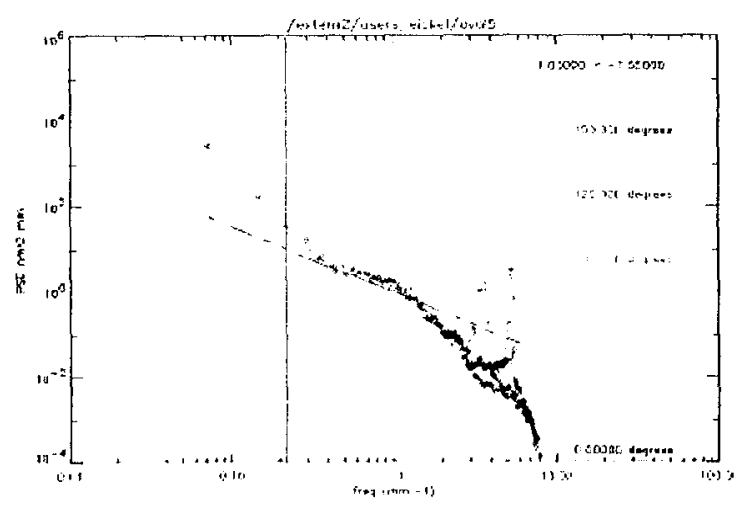

(b) 1D PSD calculated at several angular orientations. Valid region lies between the two vertical lines. 


\subsection{Thermal modeling \& cooling head design}

The parallel slab laser architecture required development of a unique cooling approach to remove the waste heat generated in the laser slabs. A cut-away view of the Mercury laser head is shown in Fig. 19. Slabs are inserted in vane elements, as shown in Fig. 20, that are stacked with flow channel gaps between them. A cross-sectional representation of the geometry is pictured in Fig. 21, depicting the three flow sections in the system. Helium cooling gas, at a pressure of $4 \mathrm{~atm}$, is accelerated in the nozzle section to a velocity of $100 \mathrm{~m} / \mathrm{s}$. The turbulent gas flow progresses down the constant area channel section removing waste heat from the laser slabs. The flow is then decelerated in the straight-walled diffuser sections downstream of the channel region. The flow system is expressly designcd to provide a well conditioned turbulent flow to the slabs with minimum potential for flow instabilities.

Optical distortions in the laser slab are controlled by three factors, listed as follows in decreasing order of importance: (1) diode source uniformity which directly translates to uniformity of the thermal source, (2) heat conduction to the colder peripheral regions of the slab assembly which enhances the temperature roll-off across the aperture, and (3) variations in the cooling flow boundary conditions associated with boundary-layer development and temperature rise in the cooling gas as it traverses the slab. Detailed thermo/mechanical/optical calculations were performed to determine the slab temperature, stress, and displacement distributions and the resulting optical distortion. The predicted phase distortion across the extracted aperture is shown in Fig. 22. A total of 0.17 waves/slab/pass is anticipated, which translates to 9.5 waves for the 14-slab, 4-pass system. The shape is low order, thereby amenable to deformable mirror correction. An important material feature of S-FAP is the negative $\mathrm{dn} / \mathrm{dT}$ coefficient, which provides for compensation of nearly $60 \%$ of the combined displacement and stress contributions to the wavefront distortion.

\subsection{Propagation \& Optical-Specifications Modeling}

We modeled the propagation of light pulses through the 4-pass architecture using the PROP92 code. ${ }^{13}$ We placed a wavefront distortion on every optical surface encountered by generating simulated wavefront distortion maps based on measurements of similar components in the LLNL Beamlet laser. This was done by computing the square-root of the magnitude of the measured optical power spectral density (PSD), multiplying by a random phase factor, and computing an inverse fast Fourier transform. For the Yb:S-FAP crystals, however, no such measurement source existed, at least at first, so we assumed that the crystal distortions would follow the fractal (i.c., powcr-law) nature observed for nearly all highly polished optics. We thereby chose to use the form $\mathrm{PSD}=\mathrm{A} \mathrm{f}^{-155} \mathrm{~nm}^{2} /\left(\mathrm{mm}^{-1}\right)$ for spatial frequency $\mathrm{f}$ in $\mathrm{mm}^{-1}$, with the 1.55 coefficient derived from measurements of essentially all of the optics on Beamlet, as represented in ID. By varying the coefficient $A$ in this expression, we calculated how the energy contained in the required Mercury focal-spot size would decrease with increasing A. To contain $\sim 100 \mathrm{~J}$, A must be $\leq 1$. This sets the optical specifications for Yb:S-FAP at an rms roughness per surface of $\leq 3.7 \mathrm{~nm}$, which corresponds to a peak-to-peak roughness maximum of $\sim \lambda / 50$, for a frequency range of $\sim 0.06$ to $\sim 40 \mathrm{~mm}^{-1}$. Other studies showed that the bulk absorption in Yb:S-FAP need only be $\leq 1 \%$ per $\mathrm{cm}$ (which is why we chose to use $0.5 \%$ per $\mathrm{cm}$ in our modeling).

Fig. 19 A cut-away view of the Mercury laser amplifier head showing the 7-slab stack of Yb:S-FAP slabs

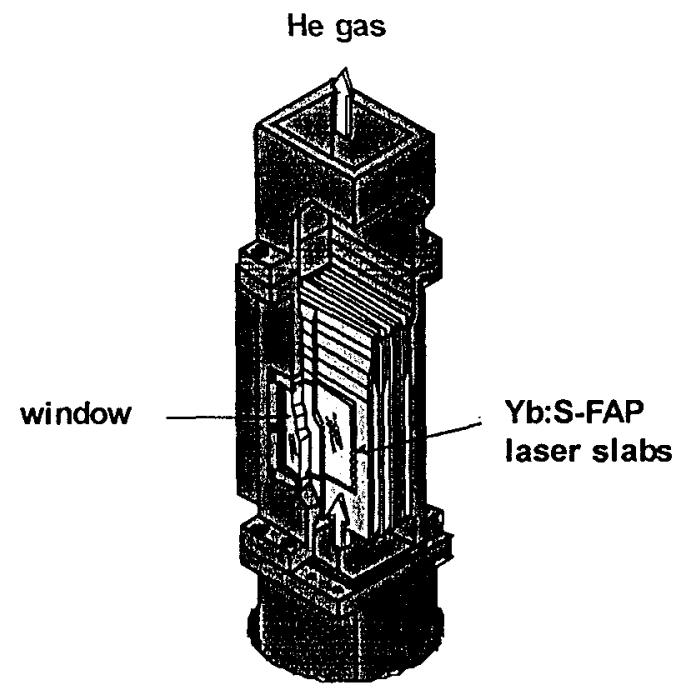




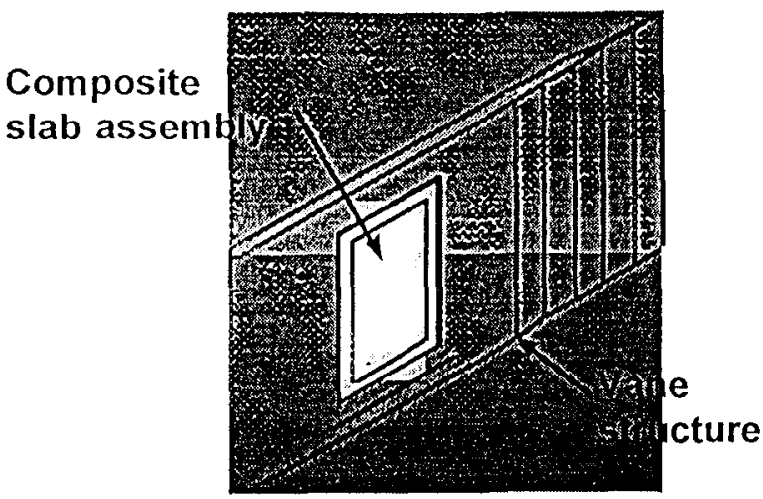

Fig. 20 -The flow vane elements for mounting the slaus (the indicated labels are "Composite slab assembly" and "Vane structure").

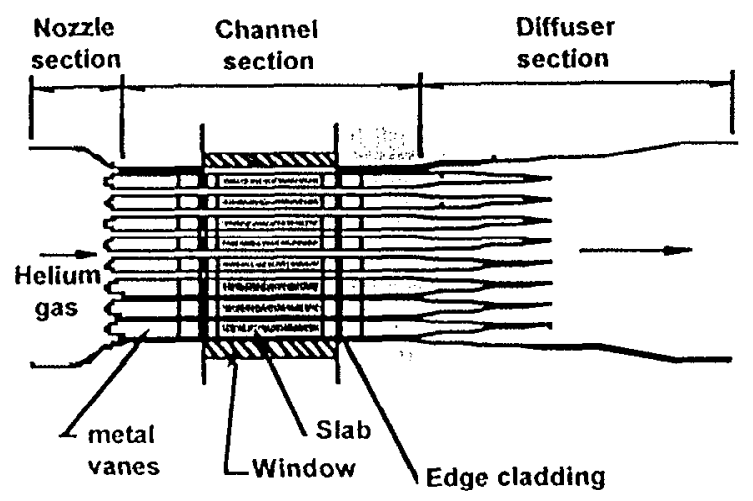

Fig. 21 -A schematic cross-section through the amplifier head showing the major components and the major flow zones

Fig. 22 The thermally induced phase distortions in a single slab. The peak distortion is 0.17 waves/slab/pass. The phase front is low order, thereby amenable to deformable mirror correction.

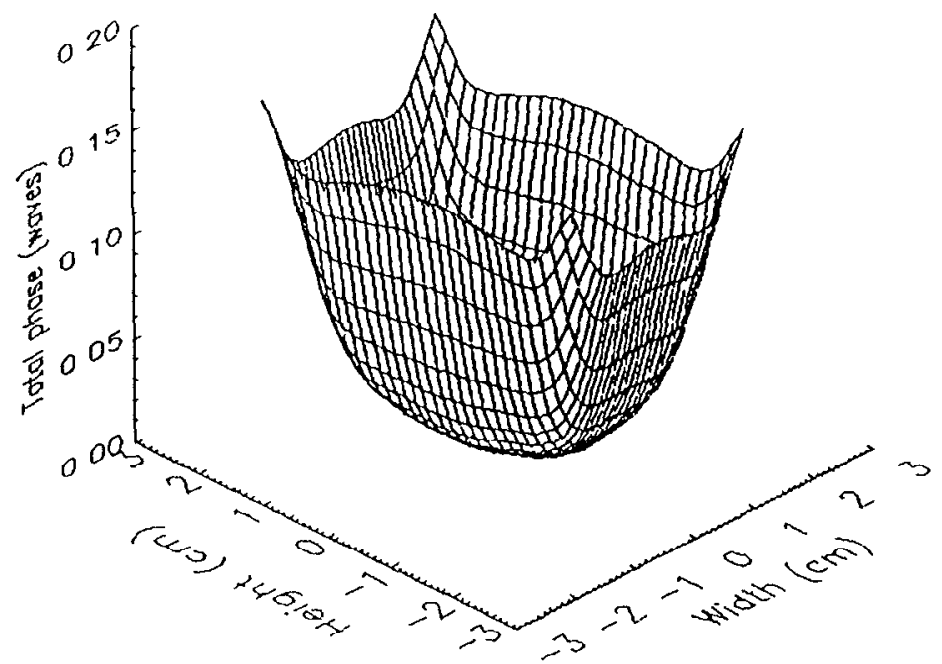




\section{CONCLUSIONS}

The Mercury laser is a significant advance in laser technology in terms of the scale of diode array peak output power, development of the largest Yb:S-FAP crystals, gas-cooled-slab architecture, and highest energy per pulse DPSSL ever built. This laser is the first serious step in developing the next-generation of high-energy-density and fusion laser systems. Our modeling of Mercury's thermal management and performance indicates that Mercury will be able to meet its performance goals. We are therefore in the process of building this 100 -joule laser.

\section{ACKNOWLEDGMENTS}

This work was performed under the auspices of the U.S. Department of Energy by Lawrence Livermore National Laboratory under Contract W-7405-Eng-48.

\section{REFERENCES}

1. C. D. Marshall, L. K. Smith, R. J. Beach, M. A. Emanuel, K. I. Schaffers, and S. A. Payne, "Diode pumped ytterbiumdoped $\mathrm{Yb}: \mathrm{Sr}_{5}\left(\mathrm{PO}_{4}\right)_{3} \mathrm{~F}$ laser performance," IEEE J. Quantum Electronics, 32, \#4, p. 650, 1996 (and ibid., 1995 ASSL Conference Proceedings).

2. C. D. Marshall, L. K. Smith, S. Sutton, M. A. Emanuel, K. I. Schaffers, S. Mills, S. A. Payne, W. F. Krupke, and B. H. T. Chai, "Diode-pumped gas-cooled-slab laser performance," OSA Trends in Optics and Photonics on ADVANCED SOLID STATE LASERS, Vol 1, Ed. S. A. Payne and C. R. Pollock, Topical Meeting January 31 - February 2, 1996, San Francisco, CA, Optical Society of America, pp. 208-212, 1996.

3. C. S. Vann, et al., "Testing a New Multipass Laser Architecture on Beamlet," ICF Quarterly Report, UCRL-LR-10582195-2, Lawrence Livermore National Laboratory, Livermore, CA, p. 142, Jan-Mar 1995.

4. Eric C. Honea, Jay A. Skidmore, Barry L. Freitas, Everett Utterback and Mark A. Emanuel, "Modeling the effect of heatsink performance in high-peak-power laser-diode-bar pump sources for solid-state lasers", Proceedings of SPIE Conference 3285B, 1998 (in press).

5. M. Voss, C. Lier, U. Menzel, A. Barwolff and T. Elsaesser, "Time-resolved emission studies of GaAs/AIGaAs laser diode arrays on different heat sinks", J. Appl. Phys. 79, p. 1170, 1996.

6. K. I. Schaffers, A. J. Bayramian, C. D. Marshall, J. B. Tassano, and S. A. Payne, "Analysis of $\operatorname{Sr}_{5-x} \mathrm{Ba}_{\mathrm{x}}\left(\mathrm{PO}_{4}\right)_{3} \mathrm{~F}: \mathrm{Yb}^{3+}$ crystals for improved laser performance with diode pumping," Advanced Solid-State Lasers, Twelfth Topical Meeting, Technical Digest, January 27-29, 1997, Orlando, Florida (Optical Society of America), p. 292/WD3-1, 1997.

7. J. J. Snyder, P. Reichert, and T. M. Baer, "Fast diffraction-limited cylindrical microlenses," Appl. Opt. 30, pp. 27432747, 1991.

8. R.J. Beach, M.A. Emanuel, B.L. Freitas, J.A. Skidmore, N.W. Carlson, W.J. Benett, and R. W. Solarz, "Applications of microlens-conditioned laser diode arrays," in Micro-Optics/Micromechanics and laser Scanning and Shaping, M.E.

Motamedi, and L. Beiser, eds., Proc. SPIE 2383, pp. 283-297, 1995.

9. R.J. Beach, "Theory and optimization of lens ducts," Appl. Opt. 35, pp. 2005-2015, 1996.

10. Raymond J. Beach, "Optimization of Quasi-Three Level End-Pumped Q-Switched Lasers," IEEE J. Quantum Electronics, 31, \#9, p. 1606, Sept. 1995.

11. See for example, Chaiyu Ai and James E. Wyant, "Measurement of the inhomogeneity of a window," Optical Engineering, 30, \#9, pp. 1399-1404, 1991.

12. J.K. Lawson, D.M. Aikens, R.E. English, Jr., C.R. Wolfe, "Power spectral density specifications for high-power laser systems," SPIE Proceeding 2775-Specification, Production and Testing of Optical Components and Systems, pp. 345-356, 1996.

13. R. A. Sacks, M. A. Henesian, S. W. Haney, and J. B. Trenholme, "The PROP92 Fourier Beam Propagation Code," ICF Quarterly Report, UCRL-LR-105821-96-4, Lawrence Livermore National Laboratory, Livermore, CA, p. 207, July-Sept. 1996. 


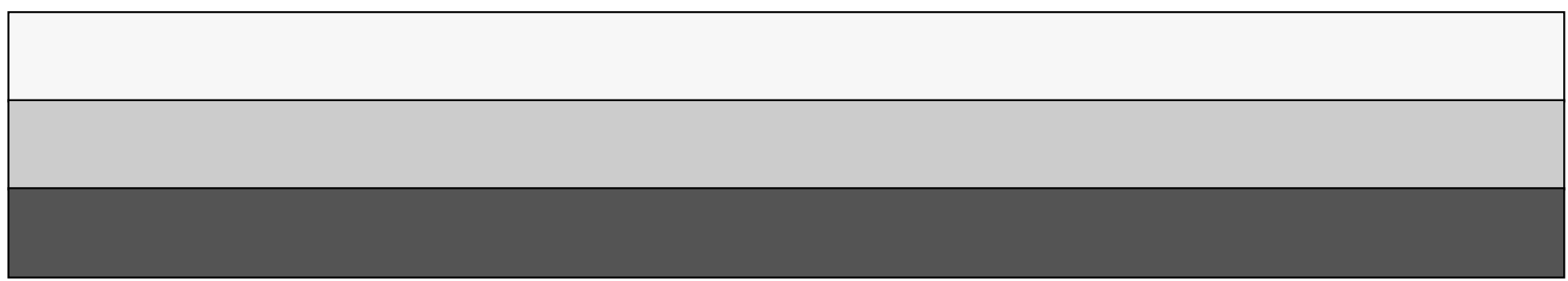

